https://helda.helsinki.fi

\title{
Moderately naturalistic metaphysics
}

\section{Morganti, Matteo}

2017-07

Morganti , M \& Tahko, T E 2017 , ' Moderately naturalistic metaphysics ', Synthese , vol.

194 , no. 7 , pp. 2557-2580 . https://doi.org/10.1007/s11229-016-1068-2

http://hdl.handle.net/10138/225345

https://doi.org/10.1007/s11229-016-1068-2

acceptedVersion

Downloaded from Helda, University of Helsinki institutional repository.

This is an electronic reprint of the original article.

This reprint may differ from the original in pagination and typographic detail.

Please cite the original version. 


\title{
Moderately Naturalistic Metaphysics
}

\author{
Matteo Morganti (Roma Tre) \& Tuomas E. Tahko (Helsinki)
}

Synthese (2017) 194: 2557-2580

\section{Abstract}

The present paper discusses different approaches to metaphysics and defends a specific, non-deflationary approach that nevertheless qualifies as scientifically-grounded and, consequently, as acceptable from the naturalistic viewpoint. By critically assessing some recent work on science and metaphysics, we argue that such a sophisticated form of naturalism, which preserves the autonomy of metaphysics as an a priori enterprise yet pays due attention to the indications coming from our best science, is not only workable but recommended.

\section{Introduction}

The debate concerning the methodology of analytic metaphysics and, in particular, the relationship between metaphysics and empirical science is currently as intense as ever. A key question in this debate is whether or not metaphysics and science share any common ground either in their methods or their subject matter. This question of course assumes that there is sufficient unity in the respective subject matters of metaphysics and science in the first place, which is by no means obviously the case. While an exhaustive discussion is impossible here, we think a sufficient amount of unity can be achieved by making the following assumptions and considerations. On the one hand, those disciplines that we currently group under the label 'science' do appear to share a common methodology, i.e., a methodology based on the formulation of hypotheses and empirical testing, and a common subject matter, i.e., the nature of the concrete world ${ }^{1}$. As for metaphysics, the preliminary conception of metaphysics that we will be dealing with has as its central task the study of ontological categories and dependence/priority relations among them. It is often thought that the

\footnotetext{
${ }^{1}$ Abstract disciplines such as mathematics are likely to constitute an exception, given their a priori nature and their object of study. We will ignore them here. Apart from this, our focus in what follows will be on the natural sciences, especially physics, but this is merely due to personal preferences and expertise. In particular, we do not wish, nor need, to formulate any verdict here concerning the methodological continuity or discontinuity between the natural and the social sciences. It may well be that all of our claims equally apply to the natural and the social sciences.
} 
study of such categories and relations is somehow prior to other areas of philosophy as well as science. We wish to take no strong stand on the priority issue right now, but it's clear that this priority assumption is grounded on two main elements that are plausibly regarded as unifying traits of the metaphysical enterprise under consideration: i) the generality of the concepts employed by metaphysicians and of the questions they ask, and ii) the a priority of their methods: they do not, at least not primarily, rely directly on empirical data. This is the dichotomy that we will be concentrating on. To begin with, then, focusing on methods and subject matter one obviously obtains the following fourfold table regarding the relationship between science and metaphysics:

\begin{tabular}{|l|c|}
\hline $\begin{array}{l}\text { No overlap regarding methods or subject } \\
\text { matter }\end{array}$ & Overlapping methods, distinct subject matter \\
\hline Overlapping subject matter, distinct methods & Overlapping methods and subject matter \\
\hline
\end{tabular}

In view of the foregoing considerations, one may invoke the traditional a priori/a posteriori distinction and consequently argue that, if at all, there is only some degree of dispute concerning the two options on the left-hand side of the table. However, that science and metaphysics can easily be separated along the a priori/a posteriori distinction has been questioned in some recent work, and we will go along exactly this route in what follows. This is not to say that the above rough indication of the unifying traits of the natural sciences on the one hand and of metaphysics on the other should be dropped. Rather, the point is that such an indication leaves the question concerning the precise nature of the relation between science and metaphysics completely open. An accurate analysis may show that there is indeed a clear demarcation between science and metaphysics, and that it corresponds to the a priori/a posteriori distinction; but it may also lead to the conclusion that a radical revision of the traditional characterisation of either science or metaphysics, or both, is required. Be this as it may, an accurate analysis is indeed in order. 
Given the fourfold table above, one gets four initial contenders²:

1) Metaphysics conceived as an independent, purely a priori discipline and science as an independent, purely a posteriori discipline, with no overlap in their methods or subject matter. One version of this position could be the view of metaphysics as conceptual analysis, focused on clarifying certain basic notions and primarily based on linguistic considerations. On this construal, metaphysics employs armchair methods, largely independent of a posteriori elements, whereas science is based on pure empirical research, without any need for armchair methods;

2) Metaphysics conceived as telling us something about the nature and structure of reality, perhaps in conjunction with empirical science, but distinct from it in terms of its methods, in virtue of its being an essentially a priori enterprise. Thus, metaphysics and science share their subject matter, but not their methods;

3) Metaphysics conceived as having a distinct subject matter from empirical science, such as purely conceptual issues, but overlapping with the latter in its methods. This means to consider metaphysics as a sui generis special science, without any distinct armchair methods, but such that it applies scientific methodology to a peculiar, well-defined domain of inquiry. So-called ‘experimental philosophy’ might subsume something of this sort;

4) Metaphysics conceived as fully 'naturalistic', i.e., only of value if it is empirically tractable if not in the sense of making predictions that can be tested by empirical means, at least in the sense of being directly grounded in scientific observation and theorising. On this view, metaphysics effectively becomes identical with science both in its methods and its subject matter. That is, metaphysical questions are either discarded as pointless, or

\footnotetext{
${ }^{2}$ We will ignore here clearly implausible candidates such as, for instance, the view that both science and metaphysics are entirely a priori in their methodology.
} 
reduced to scientific questions (but perhaps, as we will see, metaphysics is able to add something more, e.g., theoretical unification, to extant scientific hypotheses).

Our own view is that it is the second approach that holds most promise. In our view, metaphysics and science are both in the business of examining and explaining reality, even though the means by which they do so differ. Importantly, however, we also aim to show that the non-overlap of methods does not entail that science and metaphysics are two completely independent ways of asking questions about a common subject and providing answers to those questions. To the contrary, we believe the supporters of the methodological autonomy of metaphysics can, and indeed should, nevertheless insist that metaphysics ought to seek an at least indirect connection with reality through the empirical methods of science. In view of this, it is the second option above that we will focus on, and try to characterise in some detail, rather than discussing the various options in detail. In particular, we will argue in favour of what we call 'moderately naturalistic metaphysics', whereby the a posteriori and the a priori element are not subordinated one to the other, and instead enter a relation of mutual support and complementarity.

The first of the above approaches - at least in its extreme form where metaphysics is considered a pure a priori discipline - surely had some historical, authoritative proponents, but has fallen out of fashion in recent times exactly because of its 'armchair methodology'. Indeed, it seems part and parcel of contemporary western culture that we can gain knowledge of the world around us only on the basis of empirical inquiry and a posteriori reflection (be it together with other factors or not); and this idea has recently found increasing approval within the philosophical community. As for the third approach, we believe there is a lot to be learnt from studies of the sort proposed by, for instance, Alvin Goldman (see, e.g., Goldman 2007 and 2015). However, we also believe that those studies contribute to the understanding of the psychological and cognitive basis of certain categories of thought, rather than to the formulation of full-blown hypotheses and theories about reality based on those categories (or other, less entrenched ones). Thus, experimental metaphysics intended as metaphysics naturalized through the tools of cognitive science does not represent a truly viable answer to the question concerning the relationship between science and metaphysics as it is intended here. 
We thus get to the fourth perspective above, considering only empirical inquiry and a posteriori reflection relevant, and reducing the autonomy of the scope of metaphysics to either zero or to a merely pragmatic contribution in terms of unification or something similar. This view has become the most influential perspective on analytic metaphysics, at least among the most explicitly scientifically-minded philosophers, such as James Ladyman and Don Ross. And it also seems that this 'naturalisation' of metaphysics is proving very fruitful in terms of programmes of research and results (allegedly) corroborated by our best current science. In view of this, it would seem that the task of defining a plausible form of metaphysics has already been accomplished, and that fully naturalised metaphysics is the way to go.

However, things are not so simple. As recent work along these lines shows (e.g., Ladyman and Ross 2007), naturalism understood in this sense sets rather strong constraints on what counts as admissible metaphysics. As a consequence, the position is in effect only minimally different - or so we will argue from that endorsed by more radical empiricists such as, for instance, van Fraassen, who go as far as rejecting metaphysics altogether in favour of science. In particular, once one adopts the naturalistic viewpoint just mentioned, i.e., once only a posteriori elements are deemed important, the question naturally arises whether the latter, eliminative stance is not more coherent overall, metaphysics apparently playing no real role in our inquiry into the nature of reality. But if this is the case, we are also entitled to ask whether there is another, less deflationary stance available to those willing to pay due attention to science without belittling the import of metaphysics. After all, talk of overlap by no means coincides with talk of identity. In view of its popularity and of the fact that its proponents are often the loudest critics of metaphysics as a discipline distinct from science either in its methods or subject matter, it is mostly this fourth approach that we will contrast our proposal with in the rest of this paper.

The moderately naturalistic alternative we have in mind - which, as we will show in what follows, bears interesting connections with some recent proposals - is essentially built upon three ideas ${ }^{3}$ : first, that there are important methodological similarities between metaphysical and scientific modelling, and metaphysics essentially differs from science only in its greater generality and perhaps conceptual priority;

\footnotetext{
${ }^{3}$ Besides the views we will discuss, Nolan (2015) has recently put forward an interesting analysis of armchair methods as a posteriori rather than a priori.
} 
second, that metaphysics is nevertheless primarily an a priori discipline, with sui generis, irreducible features; third, that science represents at least an indirect 'testing ground' for metaphysical hypotheses, which thus get fleshed out, as it were, in the same process that employs them to provide an interpretation of our best scientific theories. It is these three ideas together, we will argue, that make room for the view that the development of metaphysics should proceed independently of - although in parallel with - that of science. These ideas distinguish our variety of moderate naturalism from traditional naturalism, as the former doesn't recommend the naturalisation of metaphysics itself, but rather the close development, and mutual sustenance, of metaphysics and empirical science. ${ }^{4}$

We develop our first suggestion in the second section, indicating those aspects of the science/metaphysics analogy based on modelling that we are sympathetic to, and where we think something must be added. We then proceed to do the same with the second suggestion, concerning the autonomy of metaphysics, in the third section. On this basis, we then look at some more deflationary, or even eliminativist, recent proposals in the fourth section, with special attention to Ladyman and Ross (2007). We argue that these approaches sacrifice too much of metaphysics, and fail to do justice to all the aspects of (dis)similarity between science and metaphysics. In the fifth section, we put forward our own view, according to which, as mentioned, the methods and subject matter of metaphysics are significantly different from those of science and yet the two activities can and should be pursued together, in such a way that metaphysics grounds the interpretation of science and, at the same time, science allows one to critically assess the available metaphysical models and select some of them over others (our third key suggestion above). At the end of the paper, a case study is briefly presented, and a concluding section follows.

\footnotetext{
${ }^{4}$ In connection to this, it is important to stress again that we will not pursue other lines of argument that have been pursued with a view to defending some 'liberal' or moderate form of naturalism: in particular, we will not deal here with considerations concerning abstract entities, normative concepts or items having to do with consciousness/subjective experience. Although the case against the more radical versions of naturalism can certainly be made stronger by pointing at the peculiarity of mathematical knowledge, the seeming irreducibility of the normative, or the depth of the so-called 'hard problem' of consciousness, we think a good case for non-radical naturalism can already be made at the level of the typical objects of inquiry of, say, physics or chemistry. More generally, our contribution is intended to be to the discussion concerning methodological, not ontological, naturalism.
} 


\section{The continuity of scientific and metaphysical methodology}

One well-argued version of the suggestion that metaphysics and science have effectively the same methodology, but distinct subject matters - hence an example of approach 3) above - is due to L.A. Paul (2012). Beginning from the second part of the claim, Paul conceives of the subject matter of metaphysics as ontologically prior to science, in the following sense:

The ontological account describes the metaphysically prior categories and constituents of the physically fundamental entities, and in this sense describes features of the world that are more fundamental than those of natural science (Paul 2012; 5.)

Crucially, Paul thinks that this ontological priority is reflected by conceptual priority. For instance, she argues that

The fact that the subject matter of metaphysics can be ontologically prior to the subject matter of science is reflected in the fact that many concepts of metaphysics are conceptually prior to the concepts of science. [...] There is no way to make sense of the central concepts of classical field theory or quantum chromodynamics without using a concept of property (Ib.; 6.)

We think that one must be careful here. It is uncontroversial that the notion of property is conceptually prior to that of, say, electric charge: electric charge is a specific case of property. In this sense, metaphysical concepts do appear more fundamental than scientific concepts. However, this, in itself, by no means indicates that metaphysical inquiry has a privileged role to play when it comes to studying the fundamental structure of reality, as one could interpret Paul to be suggesting - the sense of fundamentality at play here is not entirely clear. 
In general, conceptual priority is not a convincing criterion of ontological priority. There are at least two good reasons to think so. Firstly, it is often very difficult to even determine when one concept is prior to another - the order of the acquisition of concepts being of little help. Secondly, even if a specific sense of priority and dependence among concepts is granted, work is required to establish which of the seemingly more fundamental linguistic/conceptual categories latches onto objective structures of reality. Paul (Ib.; 9, 12) seems to have in mind an explanatory link broadly understood: for instance, to the extent that we speak of the intensity of the Higgs field in region $\mathrm{R}$ at time $t$, we seem to be ipso facto committed to the existence of a substance with a property and of a space-time background, and the latter concepts are necessary in order to understand and account for the nature of Higgs fields. However, this cannot be all there is to it. For, the mere use of certain concepts and categories as explanatorily basic should not be considered sufficient for ontological commitment - not, at least, until all alternatives have been entertained and assessed. The reason for this is that certain presuppositions concerning the ontological structure of reality (in the present example, that there is something like a substance, that it is distinct from space and time etc.) are likely to be at work in every explanation; and different presuppositions might well lead to different explanations and different ontological commitments, which clearly blocks the inference [concept usage $] \rightarrow$ [reality of the 'ontological items' corresponding to those concepts]. Thus, the priority of metaphysical concepts and hypotheses may be granted in terms of generality, but this says absolutely nothing about the grounds (if any) that we have to assert that certain metaphysical entities or processes exist (or do not exist). As a consequence of this, the distinctness of metaphysics and science at the level of subject matter can be granted, but remains vague in the context of Paul's proposal. Additionally, and relatedly, the supposedly fundamental metaphysical notions often, if not always, lend themselves to further analysis and questioning. What is a substance? Can the fundamental properties be properties of space-time directly? Is it necessary to postulate properties as belonging to an independent category? This clearly points to the fact that one must be careful when moving from metaphysical explanation to ontological commitment, and confirms the fact that Paul's claim of priority must go hand in hand with a careful definition of the scope, methodology and criteria for theory-choice that characterise metaphysics. Moreover, it must be made clear what sense of priority/fundamentality is doing the work - we have just argued that mere conceptual priority won’t do. Lacking all this, a more reductive, or even an eliminativist, view of metaphysics remains a live 
possibility - for, clearly, one may argue that, ultimately, seemingly fundamental metaphysical notions are in fact grounded in squarely scientific ones.

Let us, then, set aside Paul's claim of ontological priority for the moment, and look at another important aspect of Paul's proposal: that is, that metaphysics relies on modelling. Specifically, Paul suggests that - as in science - models are the metaphysician’s primary tool of theory-forming. Of course, according to Paul the categories of entities involved in metaphysical models are different from those used in science (e.g., one would talk of properties or substances rather than of particles or genes), but metaphysics and science both model parts of reality nonetheless. Moreover, both science and metaphysics use a priori reasoning to infer to the best explanation, which helps us choose between empirically equivalent models. ${ }^{5}$ Also, says Paul, in metaphysics as in science, the usual theoretical virtues of simplicity, ontological parsimony, elegance, explanatory power and fertility may be used to evaluate these models (Ib.; 21). Now, the idea of metaphysics as modelling is indeed attractive to us, and we agree that the sort of things metaphysicians and scientists include in their models are not the same. However, although we also share with Paul the view that inference to the best explanation and theoretical virtues play an important role, we still have some reservations about Paul's suggested strategy for choosing between empirically equivalent models - which clearly has a bearing on Paul's claim of methodological overlap between science and metaphysics. We fear that the extent of this overlap may be exaggerated.

First of all, Paul's strategy appears to be similar to that of many other authors, namely, a 'vaguely Quinean' methodology of inference to the best explanation based on quantification over the best available theories (although Paul does not mention Quine, see instead Sider 2011). This viewpoint is, as is wellknown, problematic: Does it provide a reliable link between one's metaphysical theory and the actual structure of reality, or merely a pragmatic criterion? Sider, for one, goes for the latter alternative:

\footnotetext{
${ }^{5}$ For instance, Paul says that "After a theory is selected from the mix as providing the best explanation, if one is a scientific realist, its class of models is supposed to give us the truth about the nature and structure of certain features of the world: i.e., we accept the theory as a representation of these features of the world” (Paul 2012; 12.)
} 
The Quinean thought about ontology is sometimes put in terms of indispensability: believe in the entities that are indispensable in your best theory. The analogous thought about ideology may be similarly put: regard as joint-carving the ideology that is indispensable in your best theory. This is fine provided "indispensable” is properly understood, as meaning: “cannot be jettisoned without sacrificing theoretical virtue” (Sider 2011; 14.)

Now, the strength of the indispensability intuition can be questioned (e.g., Melia 2000). Especially so if one is dealing with metaphysical rather than scientific models. And even more so if the relevant model is selected merely on the basis of pragmatic considerations, which leads us to a second, related worry.

It is widely acknowledged that, say, simpler or more elegant scientific theories need not be true, and indeed the plausibility of realist commitments based on considerations of simplicity etc. is an object of intense dispute in the philosophy of science. In science, however, empirical data can be brought to bear on the evaluation of one's conjectures - something that Paul surprisingly ignores or, at any rate, takes for granted, even though it represents a potential point of significant demarcation between science and metaphysics. Indeed, detractors of the autonomy of metaphysics (e.g., Ladyman (2012)) have argued exactly that, since in metaphysics truth is all that matters (metaphysics has no practical application) but theoretical virtues are not truth-conducive, the fact that metaphysical models can only be assessed on the basis of their theoretical virtues goes to show that it is pointless to do a priori metaphysics. ${ }^{6}$ That is, that there is no option other than extreme naturalism for the serious defender of metaphysics.

And there is more. Even setting aside the dynamics of model-selection, there are open questions concerning the very nature of metaphysical modelling on Paul's construal. A natural way to proceed to understand metaphysical modelling, Paul says, is by examining examples of metaphysical modelling, thought experiments in particular (Paul 2012; 12 ff.). Paul considers abstraction and idealization to be central in this sense. For instance, when examining the connection between two distinct events, Socrates' death and his drinking hemlock, we may abstract away from the complex details of the actual world in order to identify a

\footnotetext{
${ }^{6}$ In science, instead, whether or not one believes in the link between empirical success and truth, the former is an unquestionable fact and a fundamental guide in theory development and theory-choice.
} 
counterfactual dependence relation between these events. Paul suggests that by ignoring superfluous details, we can conclude that such counterfactual dependence is sufficient for causation. Hence, Socrates' death was caused by his drinking the hemlock. The process of abstraction is important, Paul says, because, in actuality, superfluous features may 'muck up' the real-world dependence facts. However, the model we get by abstraction is sufficiently isomorphic with the real-world case for a reliable connection, or so the story goes. If this were correct, the suggested parallel between science and metaphysics would indeed hold: in both cases, via abstraction and idealisation we get to identify the fundamental features of reality. We don’t think that this is entirely convincing, though.

This type of story about the role of counterfactuals in metaphysical modelling has been developed in detail by Williamson (2007), with whom Paul (2012; 23) explicitly agrees. But the account has its caveats: it gives us no reliable means to explain essentialist knowledge, i.e. Williamson's 'constitutive facts'. Constitutive facts are things that we must hold fixed across metaphysically possible counterfactual scenarios, and knowledge of such facts is needed to secure traditional examples of metaphysical necessities. But how is one to determine which facts are constitutive? If one's evaluation of counterfactual scenarios depends on what is held fixed, it obviously follows that there is more to metaphysical model-selection than counterfactual reasoning: at least, it also includes a non-counterfactual determination of the fixed background. The upshot is that counterfactual supposition is not a reliable epistemic guide to metaphysically necessary facts, as knowledge of constitutive facts must precede the counterfactual account itself (on this, see Roca-Royes 2011 and Tahko 2012.) $)^{7}$ It follows that Paul's claim about the methodological dimension of the science-metaphysics divide is also in need of further elaboration. In conclusion, on a closer analysis, it appears that there are aspects of metaphysical modelling which cannot be reconciled with scientific modelling.

Summarising, we take Paul to identify an important parallelism between scientific and metaphysical methodology: they both employ inference to the best explanation and criteria for theory-choice based on theoretical virtues; and they both work with abstract and idealised models of reality. There is some

\footnotetext{
7 Consider the following example. Paul argues $(2012 ; 15)$ that a counterexample to the claim that causation is necessarily a relation of counterfactual dependence between events would require finding a metaphysically possible world with a case of causation between events not exhibiting counterfactual dependence. But how can we (fail to) find such a world if whether it exists at all depends on what we take to remain fixed across possible worlds?
} 
room for separation here, though: it may be that the theoretical virtues are (and should be) weighted differently in science and metaphysics, which would lead to subtle differences in the manner in which inference to best explanation is used in the two disciplines. We are sympathetic to the idea that metaphysical models are in some sense more fundamental than scientific models, but only in the relatively innocuous sense that they are constructed on the basis of more general and encompassing concepts and categories. We definitely do not think that such greater generality corresponds in any obvious way to a 'privileged access' to something like the fundamental structure of reality. If anything, there are reasons to be suspicious of this and similar claims. Further, we think that more needs to be said on (at least) two, related counts: how metaphysical models connect to scientific models, and then to reality; and how (if at all) theoretical virtues can be good guides for theory choice in metaphysics, given the latter's (alleged) lack of connection with the empirical domain.

In what follows, we will attempt to complement Paul's account of modelling by making two basic claims: first, metaphysics is a fundamental study of possibilities, aiming to arrive at essences which are not accessible, or at any rate identifiable, on a purely a posteriori basis, nor purely a priori (at least in the case of concrete objects). Secondly, it is not the case that theoretical virtues are all there is to metaphysical 'modelchoice'. For, it is at least in some cases possible to test metaphysical hypotheses empirically, albeit in an indirect sense. This can be done by applying such hypotheses to the interpretation of our best scientific theories. We take this to improve as needed on Paul's proposal (and others we will refer to later), and more generally to provide the basis for a viable form of moderate naturalism about metaphysics.

\section{The discontinuity of scientific and metaphysical methodology}

For our present purposes, it is useful to consider next Lowe's approach to metaphysics, which we take to offer at least some of the elements that we found to be missing from Paul's proposal - while being usefully complemented by the latter in other respects. Lowe considers metaphysics and science to differ both in terms of their subject matter and with respect to methodology (and would hence be closest to a version of approach 1) as listed in the introduction). For example, he says: 
Metaphysics and empirical science are not 'continuous' with each other in any sense which implies that they have the same goals and methods, or that metaphysics is just the extension of empirical science to questions of greater generality than any that are addressed by the so-called 'special' sciences. Rather, when both are conducted fruitfully, metaphysics and empirical science exist in a symbiotic relationship, in which each complements the other (Lowe 2011; 101-102.)

Lowe's claim of complementarity is one we fully agree with. However, we disagree with Lowe with respect to the ways in which such complementarity should be translated into philosophical practice. In order to expand on this idea of complementarity in the way we intend to, the aspect of Lowe's view that we would like to focus on first is the idea that metaphysics is primarily concerned with a priori arguments for the possibility of certain ontological categories and hypotheses; and also, on partly empirical grounds, with providing arguments for the actuality of some of those possibilities (Lowe 2011; 105). ${ }^{8}$

A first important issue concerns the status of metaphysical modality, i.e., the nature of possibility. To begin with, Lowe rejects the conceptualist approach of the likes of Jackson (1998). That is, he holds that the a priori arguments of metaphysics are not exhausted by conceptual analysis. Rather, Lowe thinks, metaphysical a priori arguments are the source of the type of constitutive or essentialist knowledge that the counterfactual approach familiar from Williamson - which we encountered in the previous section - seems to struggle with. In fact, Lowe's view is that 'all metaphysics is implicitly modal' (Lowe 2011; 106) and, further, that metaphysical modality is grounded in essence (as in Fine 1994). But how do we get to know these essences? The response to this question constitutes an assumption that is the core of the methodology of Lowe's metaphysics. Lowe $(2008,2012)$ specifies that the relevant epistemic process is not based on intuitions or thought experiments, but rather on direct a priori access to essentialist facts which ground modal truths. According to him, such essentialist knowledge precedes empirical knowledge about which ontological categories are actual, that is, 'essence precedes existence'. The crucial point is, it seems to us, that Lowe maintains that our epistemic access to essences is relatively unproblematic, and doesn't in fact require any specific philosophical treatment. For instance, he says:

\footnotetext{
${ }^{8}$ For Lowe's account on ontological categories, see Lowe (2006).
} 
To know something's essence is not to be acquainted with some further thing of a special kind, but simply to understand what exactly that thing is. This, indeed, is why knowledge of essence is possible, for it is a product simply of understanding — not of empirical observation, much less of some mysterious kind of quasiperceptual acquaintance with esoteric entities of any sort. And, on pain of incoherence, we cannot deny that we understand what at least some things are, and thereby know their essences (Lowe 2008; 39.)

In other words, Lowe ties knowledge of essence closely with the notion of understanding. Everyone, Lowe thinks, can successfully grasp at least some essences and hence come to know what kind of modal constraints they impose on possible ontological categories. Consider the case of the transuranic elements: many of them were only synthesised after their possible existence was determined by nonempirical means. With the help of Mendeleev's periodic table, chemists have been able to predict the existence of a number of yet to be discovered elements and to make highly accurate predictions about their properties. Later on, we were able to synthesise these elements and verify that they indeed had the predicted properties. Lowe $(2008 ; 41)$ proposes that this process would not have been possible without a prior grasp of the essences of these transuranic elements. But simply understanding what would qualify as a transuranic element of a certain type was sufficient for defining the relevant categories.

Now, it might well be the case that a priori reflection was sufficient to predict the characteristics of the missing elements in the case of the periodic table; and that the predicted characteristics were essential features of those elements. But the crucial fact must be acknowledged that such a prediction reflected (what we take to be) objective features of the world only because, for the known elements of the table, we had prior empirical acquaintance with them. Some a priori idea of what is possible and what is not is certainly needed to even start to structure empirical data conceptually. But the type of modal rationalism that Lowe puts forward seems to infer from this the priority of our grasp of essences over the gathering of empirical data and input. This is where we disagree with Lowe, and where we think naturalism can and should be pushed a bit farther. 
In fact, not surprisingly, Lowe’s most forceful examples do not concern natural kinds, but abstract objects such as sets (Lowe 2008; 37.) In these cases, Lowe’s take on essences might well be a defensible approach. But since knowledge of natural kinds (understood as explaining what is shared by the concrete members of a kind rather than as pointing to abstract universals) requires input from the empirical sciences, the process of coming to know essences cannot be completely a priori. Indeed, even granting the controversial assumption that talk of natural kinds is unproblematic in science ${ }^{9}$, mere a priori work with concepts cannot be sufficient for producing the list of alternative models that may reflect reality. The empirical input of science plays a crucial role in substantiating any relevant classification or categorisation that aims to be more than a simple conceptual exercise. While Lowe doesn't deny this, his claim that science can determine which of the possibilities identified by metaphysics is actual falls short of constituting a satisfactory methodological basis, exactly because it seems to allow for totally unconstrained metaphysical theorising that, nevertheless, somehow latches onto reality. As a matter of fact, due to lacking the empirical element (and the fact that Lowe wants to steer clear of radical modal rationalism ${ }^{10}$ ) Lowe's point runs the risk of being reduced to an uncontroversial, but relatively unimportant, claim about our understanding of concepts (rather than grasp of 'real' essences) - much in the spirit of the linguistic version of the first approach to metaphysics that we mentioned in the introduction and that has already been ruled out as unsatisfactory by most authors in the more or less recent past, including, it would seem, Lowe himself. Alternatively, there is the risk of reducing metaphysics to a mere exercise in logical space with no indication of how the results should be put into connection with the reality they are intended to model. Summing up, although Lowe's essentialist strategy works well in certain cases, such as those dealing with mathematics and perhaps physics, it is difficult to expand the account across the board, e.g., to biology, where the essentialist line has been subject to ample criticism.

Can Lowe’s suggestion concerning metaphysics being an exploration of a sui generis, fundamental space of possibilities be saved in spite of the shortcomings just pointed out?

\footnotetext{
${ }^{9}$ As rightly noted by a referee, it is in fact undeniable that outside of mathematics and physics scientific definitions in terms of essences are often hard to come by.

${ }^{10}$ For instance, Lowe rejects the idea that there is a special form of metaphysical intuition of essences (see, e.g., Lowe 2014).
} 


\section{Empirical testing for metaphysics?}

So far, we have obtained mainly negative results: in spite of the appeal of the idea of metaphysical modelling based on inference to the best explanation and of the view of metaphysics as a study of possibilities and putative essences, in particular, no way has been identified yet in which metaphysics might fruitfully proceed as an irreducibly autonomous discipline which nevertheless is able to convey information about the real world. This leads us to deal with the obvious objection that metaphysics is, in the end, simply dispensable, and there is no point in trying to defend the autonomy of metaphysics from science with respect to either its subject matter or its methodology.

This means that we must now assess the more radical approach to metaphysics, exemplified by option 4) mentioned in the introduction, which encompasses several closely related stances sharing a generally empiricist philosophical basis. Discussing these viewpoints, and trying to show why they are ultimately unsatisfactory, will allow us to introduce our positive proposal, to be outlined in the next section, also closing the circle with what has been said in the previous sections.

A currently popular, non-eliminativist version of naturalised metaphysics is defended and articulated by Ladyman and Ross (2007). Ladyman and Ross endorse a conception of metaphysics as the search for unification among scientific theories on the basis of physics. In order to achieve such unification, naturalised metaphysics, they suggest, should follow two basic principles. The 'Principle of Naturalistic Closure’:

Any new metaphysical claim that is to be taken seriously at time $t$ should be motivated by, and only by, the service it would perform, if true, in showing how two or more specific scientific hypotheses [entertained at time $t$ ], at least one of which is drawn from fundamental physics [as intended at time $t$, jointly explain more than the sum of what is explained by the two hypotheses taken separately (Ib.; 37).

And the 'Primacy of Physics Constraint': 
Special science hypotheses that conflict with fundamental physics, or such consensus as there is in fundamental physics, should be rejected for that reason alone. Fundamental physical hypotheses are not symmetrically hostage to the conclusions of the special sciences. This, we claim, is a regulative principle in current science, and it should be respected by naturalistic metaphysicians (Ib.; 44).

The second principle may appear reasonable, at least with some qualifications. We do find that the requirement of 'consensus' in the second principle is likely to cause controversy and we would insist that the principle should be explicitly supplemented with a fallibilists attitude also towards such a 'consensus'. Also, the qualification 'fundamental' should perhaps be dropped, in view of the fact that novel facts in nonfundamental physics may lead to changes in what is regarded as fundamental physics. ${ }^{11}$ At any rate, while there are certainly ways to challenge the 'Primacy of Physics Constraint', we will accept it, if only to be as charitable as possible to Ladyman and Ross. The first principle, instead, we find quite objectionable. Why should non-eliminativist naturalists deem metaphysics acceptable only when it turns out to be useful to secure explanatory unification? Couldn't metaphysics and science enter into a mutually beneficial relationship without this producing any explanatory unification between scientific hypotheses?

Independently of this, one fundamental element in Ladyman and Ross' criticism of what they call 'neo-Scholastic' metaphysics - Lowe's work being mentioned as an example - is that it doesn't take the best available science seriously; classical physics and a commonsense representation of the world are, they claim, presupposed in most - if not all - cases. However, this is ambiguous: do contemporary analytic metaphysicians crucially rely on a caricature of science to reach their conclusions, or are simplified systems sufficient for their arguments and purposes? While Ladyman and Ross require the former interpretation to get their criticism of neo-Scholastic metaphysics going, our own anecdotal evidence suggests that the latter is

\footnotetext{
${ }^{11}$ For instance, observations of the perihelion of Mercury formed part of the evidence that led to the abandonment of Newtonian physics. We are grateful to an anonymous reviewer for the objection and the example. Again, remember that we are not interested here in issues - concerning the abstract, the normative and the contents of subjective conscious experience - that may well require solutions going against the idea of a primacy of physics.
} 
much more plausible. But rather than engage in a survey of all the recent literature, we will build our own case in the next section. A similar ambiguity emerges in connection to another criticism formulated by Ladyman and Ross, to the effect that neo-scholastic metaphysics unduly relies on the use of intuitions. To the extent that this is not a rephrasing of the previous criticism, i.e., a complaint concerning the use of commonsense beliefs in one's theorising, it is not exactly clear what the point is. On the one hand, nonexplicitly-argued-for premises are, eventually, inevitable in every piece of reasoning. On the other hand, it is clear that metaphysicians do not simply express their intuitions, but use them in the context of complex arguments. Thus, it would seem that Ladyman and Ross mistake a rhetoric/argumentative way of proceeding that metaphysics may well share with science (the role of intuition in science is far from clear, but it is not absurd to claim that scientists do not exclusively rely on empirical data and rational methods, at least in the context of discovery ${ }^{12}$ ) for a statement of intent. One complication here concerns the use of the term 'intuition', which is notoriously vague. ${ }^{13}$ But to be clear, what we mean to suggest is that both scientists and metaphysicians may use 'intuitions' at least in the sense of background assumptions influencing how they interpret (empirical) data, and perhaps even (at least implicitly) as justifications for the conclusions reached. ${ }^{14}$

Whatever one may think of this, there is another alleged issue that is really important in the present context. Ladyman and Ross support their own form of radical naturalism by claiming, among other things, that philosophers have often been wrong in deeming something possible or impossible, and it is thus best to learn directly from scientists (see, e.g., 2007; 16). More generally, one might worry (see Callender 2011) that it is unclear what the conceptual space that metaphysics is supposedly concerned with is like, and it is sensible to think that it is ultimately physical modality that determines what we regard as metaphysically possible, necessary or impossible. But are these arguments sufficient to prove the complete dispensability of the a priori element? Far from it, we claim. First of all, Ladyman and Ross' point might just reduce to a negligible observation about contingent historical facts. What actual practitioners of a discipline do or say, to

\footnotetext{
${ }^{12}$ See Tallant (2013) for an interesting discussion of the role played by intuition in physics.

${ }^{13}$ For an attempt to clarify what is at issue, see Jenkins (2014).

14 This last point is of course controversial, and one may suggest that a very important difference between science and metaphysics is constituted by the fact that scientists, unlike metaphysicians, never use intuitions for justifying their claims - rather than just reaching their formulation. Our response is that, even if we grant this difference, it doesn't follow that metaphysics is worthless, for it has not been shown that metaphysicians always use mere intuitions to justify their claim, nor, a fortiori, that they cannot but do so.
} 
begin with, is not necessarily indicative of the essential features of the discipline itself; indeed, scientific theories too, fallible though they might be, are presented as true by their 'inventors'. Scientists too have in this sense been wrong in the past. Independently of this, and more generally, if fallibility is a positive aspect of science, why not say the same about metaphysics, which would be only natural from the scientificallyminded perspective that we are assuming in this discussion?

As for the - more serious - thought that the modal space that scientists deal with is well-defined, while that of metaphysics is not, our response to it is straightforward: contrary to a tacit but widespread belief, a sensible defence of the autonomy of a priori metaphysics does not require the irreducibility of metaphysical modality. Rather, it only requires the necessity of the conceptual and methodological 'toolbox' of metaphysics for performing certain tasks. That is, if one thinks there are no strong, independent arguments for the irreducibility of metaphysical modality, one can perfectly well drop that idea and yet insist that naturalism does in any event require the use of metaphysical vocabulary. This is indeed the claim we wish to subscribe here: metaphysics is primarily characterised by the use of peculiar, irreducible concepts and categories, which are the most general concepts and categories and, exactly because of this, are (or, normally tend to be) not subject to immediate application; they are also immune to direct empirical testing.

This, together with the considerations put forward in the previous sections, appears to immediately make room for the more moderate form of naturalism about metaphysics we are seeking. In particular, it substantiates our earlier claims: i) contrary to Lowe, that essence precedes existence does not entail that a priori metaphysical analysis is (always) independent of, and prior to, empirical inquiry; and ii) even in a naturalistic context it can be accepted that metaphysics is a sui generis inquiry, aiming to explore a range of possibilities concerning the essential features of things. This is because it is reasonable to think that a very general concept (such as that, say, of 'individuality') admits, logically, of many different declinations (e.g., Leibnizian qualitative individuality, primitive individuation etc.) that can be defined and evaluated at the purely a priori level; and yet becomes truly informative only when empirical information is, as it were, plugged in (e.g., in the form of input concerning our best scientific descriptions of specific domains of entities that may or may not qualify as individuals). At the same time, we can accommodate Paul's claim that metaphysical models are in some sense more fundamental than scientific models (keeping in mind earlier 
reservations about the interpretation of ontological vs. conceptual priority). This is the case exactly because they are built on the basis of more general concepts and categories, which are distinctive of the possibility space we are talking about here (in our example, a metaphysical model describing individuals of various types will - or at any rate should - subsume more specific information about, say, physical individuals of various types, but also biological individuals, sociological individuals and so on) and cannot in any way be 'translated' into scientific vocabulary, i.e., fully accounted for, investigated and be fleshed out by empirical means (we mentioned the notion of individuality a moment ago, other examples include causation, ontological priority/dependence, parthood, diachronic identity, etc.).

It is true that, if metaphysical modality were to be fully reducible to physical modality, then our claim that the a priori is an indispensable part of the metaphysician's 'toolbox' would be weakened. For, it would become a possibility, in principle only dependent on contingent practical and epistemic factors, that the level of generality that is not inquired into by science at a given time becomes amenable to scientific inquiry at a later time. However, this does not entail that metaphysics is bound to be reduced or eliminated. As a matter of fact, it is also compatible with the possibility that there will always be something that does not lend itself to direct scientific investigation. Be this as it may, the non-reducibility of the metaphysical vocabulary now is already sufficient for our proposed form of moderate naturalism to be advisable, at least for researchers of the present. Whatever one thinks about modality, then, we think moderate naturalism about metaphysics can make do with the simple claim that (at present) the terms and concepts of metaphysics are not (fully) reducible to those of science - that is, not amenable to purely empirical explanation and investigation - and by working with these terms and concepts in an a priori fashion one can go some way towards defining possible ways the essential aspects of things might look like, with a view to then assessing these claims on the basis of indications coming from the actual world as it is described by our best science. We acknowledge that this is a claim that requires further support (the case study to be presented in the next section is intended to go in that direction). However, our primary goal here was to carve out the logical space for the moderate view that we wish to defend. ${ }^{15}$

\footnotetext{
${ }^{15}$ An interesting question that is worth mentioning at least in passing concerns the status of allegedly scientific theories which are however, not amenable to empirical testing, such as, for instance, string theory. Many thinkers do regard string theory as a piece of metaphysics (more often than not, intending this in a pejorative sense). We do agree that the
} 
This leads us to the final section of our paper, which contains a discussion of the most radical forms of empiricism/naturalism that lead to the explicit elimination of metaphysics, and to a defence of another thesis that is crucial for our view but has only been formulated in passing so far. Namely, the idea that the link between science and metaphysics is provided by the fact that the metaphysical exploration of the abovementioned sui generis possibility space is instrumental to the interpretation of scientific theories, which is in turn the basis for the 'indirect testing' of metaphysical hypotheses. This will also allow us to say more on an issue that we encountered earlier, having to do with empirical testing and theory-choice. The problem being, to briefly remind readers, that since metaphysical hypotheses are not connected in any way to the empirical domain, such hypotheses have to be evaluated merely on the basis of pragmatic/theoretical virtues, and remain irredeemably underdetermined.

Some of the radical forms of eliminativism we have in mind (as, for instance, Ritchie's 'deflationary naturalism' (2008) or Maddy’s ‘second philosophy' (2007)) do not require a particularly detailed treatment here, as they do not really present arguments against metaphysics but, rather, a specific approach to philosophy based on empirical elements only. Similarly, it is also relatively easy to dismiss the extreme view of those philosophers (see Maclaurin and Dyke 2012) who insist that metaphysics should be naturalistic in the sense that it shouldn't be in principle unable to have observable consequences. In a sense, this requirement is too vague: as McLeod and Parsons (2013) convincingly argue in response to Maclaurin and Dyke (drawing a parallel with the failure of Ayer's criterion of 'factualness'), every theory can be made to have observable consequences by aptly adding auxiliaries to it. ${ }^{16}$ In another sense, it is too strict: if it is true, as we are claiming here, that metaphysics should remain distinct from empirical science and essentially

lack of direct testability makes string theory and similar constructions strictly speaking non-scientific. However, we also stress the fact that string theory is not a fully metaphysical hypothesis either, at least on our construal. For, it lacks at least two fundamental features: first, being formulated in a non-scientific vocabulary; secondly, being at least potentially the basis for the interpretation of other hypotheses and theories that clearly qualify as scientific.

${ }^{16}$ Dyke and Maclaurin (2013) offer a response, specifying that they had in mind a much stricter sense of 'auxiliary hypothesis' than McLeod and Parsons, namely, only those hypotheses that are best supported by current science. But the upshot they draw from this is the one already familiar from Ladyman and Ross: 'non-naturalistic theories are those that, when conjoined to our best theories about the way the world is, do not make any novel predictions about what we should observe' (180). We have already addressed this type of objection above, and will get back to it shortly. The main point is that before any predictions can be made, we must be aware of and able to understand the assumptions and claims that a theory makes, and this means that non-empirical elements are also required. We mentioned the case of individuality above - what sense can we make of the claims about the individuality or lack thereof of electrons based on the results of quantum mechanics if we do not understand what individuality is? 
consist in an a priori analysis of modal space in terms of possible models, it simply shouldn’t be expected to have, by itself, direct empirical consequences.

It is instead both necessary and useful to discuss in more detail the views put forward by van Fraassen (in particular in van Fraassen, 2002), who presents a number of considerations in favour of a metaphysics-free empiricism, generally making a rather powerful case for eliminativism. Three of van Fraassen’s criticisms appear particularly important for our present purposes.

First, van Fraassen argues that the remoteness of metaphysical questions from empirical considerations makes them useless, even if not meaningless. He notes that science is constantly and harshly tested, and often falsified, but this doesn’t undermine, but rather grounds, its practical relevance. In contrast, metaphysics seeks the truth, but is never in a position to establish whether anything it says is actually true or false, and therefore turns out to be a merely formal exercise. Additionally, van Fraassen claims that metaphysical questions are irredeemably context-dependent and lack well-defined 'answering strategies' (he uses the example of the question 'Does the world exist?') Lastly, van Fraassen objects that metaphysics accounts for 'what we initially understand [in terms of...] something hardly anyone understands' (2002; 3), and consequently turns out to be a superfluous addition to the indications coming from empirical science. It is clear that, if these objections work, even granting that the metaphysical vocabulary is irreducible, there would seem to be no reason nonetheless for pursuing extra-scientific avenues of research about reality.

Starting from the last of these objections, we argue that it is simply false that scientific theories are 'initially understood', for they cannot be understood unless interpreted, and interpretation requires tools coming from outside of science (the interpretation of any set of signs S cannot be provided within S, for otherwise it should itself be interpreted, giving rise to an obviously vicious regress). Before those tools are applied, at most one has the sort of instrumental ability and knowledge that can only be deemed satisfactory on a very narrow conception of knowledge and understanding. Indeed, it seems clear to us that these instrumental abilities may be satisfactory for scientists insofar as the latter are busy formulating and testing 
theories, but cannot be satisfactory insofar as science must be understood as mapping the way the world is ${ }^{17}$ or, at any rate, be the subject for further analysis and reflection.

Now, if metaphysics turns out to be necessary for interpreting scientific theories, it also appears sensible to think that the concepts and categories typical of metaphysics - i.e., the general notions contained in the metaphysical vocabulary that we are presenting as indispensable for interpreting scientific theories are not (necessarily) obscure. Indeed, since metaphysical vocabulary has been and is used to interpret scientific theories that were otherwise indecipherable as descriptions of the world we live in, van Fraassen's argument from obscurity does not get off the ground. Moreover, metaphysical analysis appears in this respect closer to commonsense than its scientific counterpart - reference to commonsense obviously having a positive connotation in connection to van Fraassen's overall philosophical perspective. For instance, is the notion of a universal, say, any more obscure than that of a Higgs boson? The answer is by no means obviously affirmative unless one equates clarity with measurability. But, again, this is not what one normally intends by 'understandability' and 'clarity' - not even in an openly instrumentalist context such as van Fraassen's. If concepts such as substance, relation, property and the like are systematically, or in most cases, more understandable (or at least no less understandable) than scientific concepts - and are in fact employed at least in some cases to interpret scientific theories - then van Fraassen's criticism appears neutralised.

This gives us a clue for answering the other two of van Fraassen's objections, so finally closing the circle we opened when discussing Paul's views on metaphysical inquiry - and in particular inference to the best explanation and pragmatic virtues. First, since it informs the interpretation of science, metaphysics is in a sense at least indirectly testable, i.e., it is not entirely immune to, and indifferent towards, empirical input (for instance, it appears simply wrong to say that the presentist conception of time is not challenged by the

\footnotetext{
${ }^{17}$ Of course, this is something that a scientific anti-realist such as van Fraassen could deny. Notice, however, that we are not taking issue here with the overall coherence of those anti-metaphysical stances that are based on scientific antirealism - we just want to discuss the more specific claims, listed above, that van Fraassen makes about metaphysics. The acceptance of scientific realism might, we think, lend further plausibility to our arguments and/or be itself supported by those arguments. The idea is that, on the one hand, scientific realists believe that there is some structure to reality that science tracks, hence aptly naturalistic metaphysicians may hope to be after the same structure; and, on the other hand, that the definition of coherent scientific-cum-metaphysical views of reality may lend support to the basic intuition underpinning the realist stance, i.e., that there is indeed a structure of reality that we have the possibility to gain a progressively more accurate knowledge of. However, it may also be that scientific anti-realists are metaphysical anti-naturalists, or that they are naturalists about metaphysics but take metaphysical hypotheses as mere fictions. The issue is complex and we need not, hence will not, discuss this further here.
} 
empirical data, specifically, the special theory of relativity). This form of indirect testability is exactly what we take to be key to a proper characterisation of naturalised metaphysics, and what we referred to earlier, in questioning Paul's exclusive reference to theoretical virtues when defining the dynamics of metaphysical model-selection. Indeed, indirect testability, we suggest, is what enables one to build a bridge between scientific and metaphysical models and steer clear of the objection that, since metaphysics is not empirically relevant and yet aims at the truth, it should be simply dismissed. Secondly, and relatedly, the systematic application of metaphysical concepts and hypotheses (taken from the space of possibilities we mentioned earlier) with a view to interpreting our best current science seems to provide at least one criterion for going about answering metaphysical questions and selecting between metaphysical conjectures. What gets selected, in particular, are those among the latter that are (virtuous in terms of theoretical virtues and) most suited, all things considered, for making sense of the best available science. This, together with the strategy based on the modal constraints for scientific theorising discussed earlier, i.e., the idea that a preliminary exploration of possibility space is necessary for scientific inquiry to even get started, is tantamount to saying that, in spite of its autonomy, metaphysics is not a merely formal game which in principle lacks definitive answering strategies. $^{18}$

\section{Balancing metaphysics and science}

To sum up, the core of our positive proposal is that we consider some elements of metaphysics to be prior to science in that metaphysics explores a basic possibility space in such a way that the grounds for the interpretation of scientific theories are laid. At the same time, some elements of science are prior to metaphysics in that science not only contributes to the definition of the basic possibility space itself, but also gathers the indications coming from the actual world that are necessary for fleshing out the various metaphysical hypotheses and selecting the most appropriate (i.e., informative, explanatory, simple etc., but

\footnotetext{
${ }^{18}$ An interesting thing to notice in this connection is that Ladyman and Ross - in harmony with their endorsement of a non-eliminativist form of naturalism - put forward a positive metaphysical proposal in their book: so-called ontic structural realism, i.e., the view that reality is ultimately constituted by relations only. As the ongoing controversy about the strength and even meaning of ontic structural realism shows, such a conjecture is far from being a more or less direct consequence of contemporary science. In fact, it would seem that it can only be defined by first looking at the available possibility space with metaphysical - not physical, or at any rate scientific - glasses, and making choices that are by no means exclusively based on empirical data.
} 
also likely to be true) among them. ${ }^{19}$ Given this parallelism, claims about the respective fundamentality of science and metaphysics have to be re-evaluated. The picture that emerges is that the methodologies of the two disciplines, while distinct, are intertwined to such an extent that we cannot properly pursue one without the other if we want to describe and understand the structure of reality. As for their subject matter, we are happy to grant the fundamental unity and uniqueness of the domain that scientists and metaphysicians explore (or, at least, should explore) - both in terms of kinds of entities and in terms of modal structure: the target is 'just' the items that populate the concrete physical world, and the way they do so. The picture that emerges is thus a version of option 2) as listed in the introduction, with the addition of the mentioned caveats about intertwined methodologies.

Let us close by presenting a few more considerations in support of our proposed view on naturalistic metaphysics, and a brief case study. As has been argued, we find that our inquiry typically starts from certain empirical facts (ideally in accordance with the Ladyman-Ross 'Primacy of Physics Constraint') and then proceeds to more speculative areas, such as, for instance, thought experiments where alternative laws of physics are entertained. Then, the process moves back towards the empirical, when it comes to selecting the metaphysical models that are most likely to tell us something about the fundamental structure of reality, i.e., those that provide the best interpretation of our scientific theories. This, we suggest, is just as it should be: we ought to take our best science into account when we do metaphysics, but at some point we will have to go beyond it. At the same time, it is a perfectly legitimate endeavour for metaphysics to engage in an abstract analysis of (metaphysical) possibilities without seeking explicit confirmation from science, but this does not mean that such an analysis can be completely independent of science. In fact, there are areas of metaphysics where science may appear unlikely to ever offer anything more than underdetermined answers. But this fact cannot and should not be given a general normative connotation. Much like in the case of pure mathematics, it is often the development of science itself that both stimulates the development of the more abstract a priori work, and turns out to benefit from the enlarged set of conceptual tools defined by such work - thus vindicating its rationality.

\footnotetext{
${ }^{19}$ It is also worth mentioning here a possible precursor to this type of view - of metaphysics and science both engaged in a study of the possibility space - kindly pointed out to us by an anonymous referee: Hooker (1987).
} 
And now for the case study as an example of the suggested dynamics of mutual support between science and metaphysics. A basic issue concerning the nature and structure of reality that both physics and metaphysics deal with concerns the 'basic building blocks' of the world. $20^{\text {th }}$ century physics has led to the development of the so-called Standard Model of elementary particles, a very successful model based on quantum field theory, of which the recent discovery of the Higgs boson is just the latest striking confirmation. But what exactly does the Standard Model represent? Are elementary particles more or less like the individual things we interact with on a daily basis, or are they manifestations of something more fundamental? Is the Standard Model - regardless of its currently being the dominant paradigm among scientists - the only relevant alternative? In particular, is the assumption of a fundamental level of basic entities, which do not depend on anything else but which everything else depends on, truly inevitable? To these questions, physics simply does not provide systematic answers - indeed, no answer can be offered other than that represented by the model that is dominant in the scientific community of a given time, judging on the basis of which one might well conclude that atomism must be simply taken for granted.

Different metaphysical models of the structure of reality, however, have competed since antiquity. In particular, the Democritean atomistic model and Parmenides' monistic idea based on the unity of the One remained alive in philosophical thinking until the modern and contemporary era. But how is one to establish whether and why they are the only possible alternatives, and whether one of them truly captures the essential features of the world out there? Pure a priori work, it should now be clear, is simply unable to settle the matter. Here is where the mutual interplay and parallel development of science and metaphysics becomes possible, if not essential, as an alternative to the seeming impasse between the a priori and the a posteriori. And here is where, we think, our characterisation of this relationship becomes credible. In a nutshell, this is how we think (some significant aspects of) the situation could be represented:

1) Faced with the available empirical input - both of the commonsense and scientific type - metaphysicians explore a space of possibilities through distinctive a priori means, and by doing so they identify a range of general, seemingly internally coherent alternatives: besides 
atomism and monism, less 'conventional' views such as, for instance, a form of 'metaphysical infinitism' whereby there simply is no ultimate layer of reality, or a form of 'metaphysical coherentism' based on circular relations of dependence and priority ${ }^{20}$;

2) These alternatives are conceptually valuable independently of the empirical data. Indeed, like mathematical models and hypotheses, they emerge on the basis of peculiar, sui generis concepts: for instance, metaphysical infinitism as a perspective on the structure of reality emerges out of the concept of 'gunk' - expressing the possibility of something being infinitely divisible (everything having a proper part) ${ }^{21}$;

3) The input of science is nevertheless crucial in validating these new possibilities. ${ }^{22}$ In the case at hand, for instance, the idea of gunk - as the ground of infinitist models of the universe - seems to be at work in the definition of an important alternative to the Standard Model: Nobel prize winner Hans Dehmelt's (1989) model whereby an infinite series of layers of three particles each leads towards Dirac point particles in the limit. This example shows that, although they are derided by the more radical naturalistic metaphysicians/empiricist philosophers, purely philosophical concepts that apparently qualify as 'neo-scholastic' in a pejorative sense ${ }^{23}$ are (or at least may turn out to be) heuristically valuable in seeking to describe the ultimate structure of reality (and can perfectly turn to be so valuable only after their introduction in metaphysics);

4) Analogously, the abstract models of metaphysics gain credibility, or at any rate interest, insofar as they act as the basis for scientific models grounded in empirical input and testing. This does not mean that, say, Dehmelt's model was made possible by an explicit and conscious reference to a metaphysical idea; nor that the mere existence of this 'exotic' physical

\footnotetext{
${ }^{20}$ Notice that the same holds also for 'purely' metaphysical issues: for instance, speculations about universals, tropes, substrata and the like may plausibly be said to arise from reflection on everyday facts of qualitative similarity and dissimilarity.

${ }^{21}$ If the world is gunky there is at least one proper part of it that is such that every proper part of it has a further proper part.

${ }^{22}$ Which clearly entails, among other things, that 1) and 2) above do not mean that scientific inquiry comes later. To the contrary, as shown by our reference to 'scientific input' in 1), we believe that, starting from the human amazement in front of the complexity and mysteriousness of reality, practical and theoretical work get started together.

${ }^{23}$ The concept of gunk is explicitly indicated as an example of bad metaphysics, for instance, by Ladyman and Ross (2007; 20). It is interesting to notice, in passing, that they seem to employ something like it themselves when arguing for the viability of a version of 'ontic structural realism' whereby reality is 'relations all the way down'.
} 
conjecture in some sense confirms metaphysical infinitism. It does mean, though, that such a model was made possible by the realisation that a certain possibility was workable, worth consideration and also capable to account for the available empirical data. In this sense, one could say that whether infinitism was 'discovered' by metaphysicians or physicists is an otiose question - in either case this 'discovery' is compatible with the idea of a constrained possibility space which we have tried to elaborate here;

5) Once the relevant models have been identified, then one can agree with Paul that pragmatic considerations play an explicit role in physics as well as in metaphysics. Dehmelt, for instance, presents the infinite regress model as simpler than the dominant Standard Model. Besides the fit with the data, in this particular case explanatory power and compatibility with supposed physical laws are also crucial: Dehmelt's model has the advantage of avoiding the postulation of literally point-like particles, while zero-dimensionality and lack of spatial extension are normally assumed, albeit merely for predictive utility, when operating with the Standard Model. Since point particles are, strictly speaking, physical impossibilities and are consequently usually regarded as inevitable idealisations, this may count as a methodological argument in favour of Dehmelt's model and against the Standard Model. Something similar, we think, can and should happen in metaphysics, various competing hypotheses being critically compared to one another on the basis of their fit with empirical data and different scientific models, as well as in terms of simplicity, fruitfulness, coherence with other theories, and so on. So, it is at this stage of modelcomparison that pragmatic virtues become (as factors additional to empirical adequacy) crucial even though, it must be pointed out explicitly, it doesn’t look like final decisions are likely to emerge from this (which, however, is the case in metaphysics as much as in science, due to the very nature of inference to the best explanation);

6) Even if no final verdict regarding the ultimate structure of priority relations of the physical world is forthcoming, it nevertheless seems that what allows one to make the most 
progress $^{24}$ is a continuous interaction between the a priori and the a posteriori, whereby the possibilities identified via metaphysical tools are substantiated in the form of physical models, which in turn allow the former to be indirectly tested against the empirical domain. If they are accessible to us at all, then it is in this way that putative essences of things can best be expected to be arrived at.

In this interaction, we repeat one last time, at no point does the need for a metaphysical modality allegedly irreducible to nomological modality arise - nor the opposite need to reduce the former to the latter. What is important is that metaphysics and physics (more generally, empirical science) are just two distinct aspects of our attempt to discover the structure of reality - regardless of whether the latter has two or only one set of characteristic features. ${ }^{25}$

\section{Concluding remarks}

In this paper, we have articulated and defended a 'moderately’ naturalistic approach to metaphysics that we take to strike the best balance between science and metaphysics, and thus between a posteriori and a priori inquiry. We have focused on natural science and physics in particular on one hand and core areas of ontology on the other, but we believe that at least some of our results can be generalized to science and metaphysics more broadly conceived. From the more radical, empiricist perspective (ultimately leading to strong forms of deflationism if not to eliminativism), our approach took the idea that it is really empirical science that provides us with the access to essences, i.e., with knowledge of the fundamental features of

\footnotetext{
${ }^{24}$ To avoid ambiguities, we are not arguing here for the necessity of metaphysics for the progress of science (although we think there is in fact such a necessity); rather, we are claiming that the most progress in our knowledge of reality broadly understood is achieved by using the a priori tools of metaphysics and the a posteriori tools of science together rather than as exclusive alternatives.

${ }^{25}$ It goes without saying that, as argued by many in the past, the demarcation between the metaphysical and empirical elements of this methodology is not sharp, and it is plausible to think that one blurs into the other at the boundary. This does not affect our view, which relies on the fact that at least extreme cases can be sharply distinguished one from the other; and that both aspects are in any case equally indispensable, independently of how fuzzy or sharp the dividing line between the two is. (As a matter of fact, it is not difficult to see that the idea of moderately naturalistic metaphysics is, if anything, rendered more plausible by the vagueness of the dividing line between science and metaphysics.)
} 
reality - if there are any to be found. To this, however, unlike deflationists/eliminativists, we added a fundamental a priori element, which we take to be non-eliminable in all approaches that do not endorse fully instrumentalist views of science, and that makes us able to i) identify possible ways reality could be like (i.e., alternative metaphysical models claiming to represent essential features of reality) in the most comprehensive possible manner; and ii) lay the basis for the interpretation of the various scientific theories and models. In this sense, we suggested, a priori metaphysics has roughly the same level of independence as pure mathematics, and is similarly relevant for empirical science, in the sense that it is able to become more than a purely abstract exercise in possibility space. In closing, we provided a brief example of moderately naturalistic metaphysics at work, having to do with models of the ultimate structure of reality in terms of priority, dependence and (allegedly) basic constituents. The example is paradigmatic, we think, of the way in which a continuous interaction takes place between the a priori and the a posteriori (whether or not the individual scientist or philosopher is, fully or partially, aware of such interaction), and of how this is the best we could aim for when it comes to trying to gain knowledge of the fundamental features of reality - the difficulty of singling out univocal, definitive answers notwithstanding. We hope that future work will be devoted to more and more case studies at the boundary between physics (and, more generally, science) and metaphysics, and that they will take heed of the methodological guidelines that have been recommended in this paper.

\section{Acknowledgments}

We'd like to acknowledge the helpful feedback from Travis Dumsday, Donnchadh O’Conaill, and audiences at Helsinki, Hong Kong, and Singapore, where previous versions of this paper were presented. Tahko's research for this paper was supported by Academy of Finland grants no. 266256 and no. 274715. 


\section{References}

Callender, C. 2011. 'Philosophy of Science and Metaphysics.' In French, S. and Saatsi, J. (eds.), The Continuum Companion to the Philosophy of Science. London: Continuum, 33-54.

Dehmelt, H. 1989. ‘Triton, ...Electron, ...Cosmon, ...: an Infinite Regression?’ Proceedings of the National Academy of the Sciences USA, 86, 8618-8619.

Dyke, H. and Maclaurin, J. 2013. 'What Shall We Do with Analytic Metaphysics? A Response to McLeod and Parsons.' Australasian Journal of Philosophy 91, 179-182.

Fine, K. 1994. 'Essence and Modality.' In J. E. Tomberlin (Ed.), Philosophical Perspectives, 8: Logic and Language. Atascadero, CA: Ridgeview, 1-16.

Goldman, A.I. 2007. 'A Program for 'Naturalizing' Metaphysics, with Application to the Ontology of Events.' The Monist 90 (3): 457-479.

Goldman, A.I. 2015. 'Naturalizing Metaphysics with the Help of Cognitive Science.' In Bennett, K. and Zimmerman, D.W. (eds.), Oxford Studies in Metaphysics, vol. 9, 171-212.

Hooker, C.A. 1987. A Realistic Theory of Science. Albany, NY: SUNY Press.

Jackson, F. 1998. From Metaphysics to Ethics: A Defence of Conceptual Analysis. Oxford: Clarendon Press.

Jenkins, C.S. 2014. 'Intuition, “Intuition”, Concepts and the A Priori.' In Booth, A.R. and Rowbottom, D.P. (eds.), Intuitions. Oxford: Oxford University Press, 91-115.

Ladyman, J., 2012. 'Science, Metaphysics and Method.’ Philosophical Studies 160 (1), 31-51.

Ladyman, J., and Ross D. (with Spurrett, D. and Collier, J.) 2007. Every Thing Must Go: Metaphysics Naturalized. Oxford: Oxford University Press.

Lowe, E.J. 2006. The Four-category Ontology. Oxford: Clarendon Press.

Lowe, E.J. 2008. 'Two Notions of Being: Entity and Essence.' Royal Institute of Philosophy Supplements 83 (62), 2348.

Lowe, E.J. 2011. 'The Rationality of Metaphysics.' Synthese 178, 99-109.

Lowe, E.J. 2012. 'What is the Source of Our Knowledge of Modal Truths?' Mind 121, 919-950.

Lowe, E.J. 2014. 'Essence vs. Intuition: An Unequal Contest.’ In Booth, A.R. and Rowbottom, D.P. (eds.), Intuitions. Oxford: Oxford University Press, 256-68.

Maclaurin, J. and Dyke, H. 2012. 'What is Analytic Metaphysics For?' Australasian Journal of Philosophy 90, 291306.

Maddy, P. 2007. Second Philosophy: A Naturalistic Method, Oxford: Oxford University Press.

McLeod, M. and Parsons, J. 2013. 'Maclaurin and Dyke on Analytic Metaphysics.' Australasian Journal of Philosophy 91, 173-178.

Melia, J. 2000. 'Weaseling Away the Indispensability Argument.' Mind 109, 455-479.

Nolan, D. 2015. 'The A Posteriori Armchair.' Australasian Journal of Philosophy 93 (2), 211-231.

Paul, L.A. 2012. 'Metaphysics as Modeling: The Handmaiden’s Tale.' Philosophical Studies 160, 1-29.

Ritchie, J. 2008. Understanding Naturalism. Durham: Acumen.

Roca-Royes, S. 2011. 'Modal Knowledge and Counterfactual Knowledge.' Logique et Analyse 54, 537-552.

Sider, T. 2011. Writing the Book of the World. Oxford: Oxford University Press.

Tahko, T.E. 2012. 'Counterfactuals and Modal Epistemology.' Grazer Philosophische Studien 86, 93-115.

Tallant, J. 2013. 'Intuitions in Physics.’ Synthese 190 (15), 2959-2980.

van Fraassen, B. 2002. The Empirical Stance. New Haven: Yale University Press.

Williamson, T. 2007. The Philosophy of Philosophy. Oxford: Blackwell. 